\title{
Assimilating flow and level data into an urban drainage surrogate model for forecasting flows and overflows
}

Schou Vorndran Lund, Nadia; Madsen, Henrik; Mazzoleni, Maurizio; Solomatine, Dimitri; Borup, Morten

Published in:

Journal of Environmental Management

Link to article, DOI:

10.1016/j.jenvman.2019.05.110

Publication date:

2019

Document Version

Peer reviewed version

Link back to DTU Orbit

Citation (APA):

Schou Vorndran Lund, N., Madsen, H., Mazzoleni, M., Solomatine, D., \& Borup, M. (2019). Assimilating flow and level data into an urban drainage surrogate model for forecasting flows and overflows. Journal of Environmental Management, 248, [109052]. https://doi.org/10.1016/j.jenvman.2019.05.110

\section{General rights}

Copyright and moral rights for the publications made accessible in the public portal are retained by the authors and/or other copyright owners and it is a condition of accessing publications that users recognise and abide by the legal requirements associated with these rights.

- Users may download and print one copy of any publication from the public portal for the purpose of private study or research.

- You may not further distribute the material or use it for any profit-making activity or commercial gain

- You may freely distribute the URL identifying the publication in the public portal 


\title{
1 Assimilating flow and level data into an urban drainage 2 surrogate model for forecasting flows and overflows
}

\author{
3 Nadia Lund $^{* 1}$, Henrik Madsen ${ }^{2}$, Maurizio Mazzolenii, ${ }^{3, \mathrm{i}}$, Dimitri Solomatine ${ }^{3,4,5}$, and Morten Borup ${ }^{1}$ \\ 4 \\ $5 \quad{ }^{*}$ corresponding author: nalu@env.dtu.dk \\ $6{ }^{1}$ Department of Environmental Engineering (DTU Environment), Technical University of Denmark, \\ 7 Bygningstorvet, Building 115, 2800 Kgs. Lyngby, Denmark \\ $8 \quad{ }^{2}$ DHI, Agern Allé 5, 2970 Hørsholm, Denmark \\ $9 \quad{ }^{3}$ IHE Delft Institute for Water Education, Delft, The Netherlands \\ $10 \quad{ }^{4}$ Water Resources Section, Delft University of Technology, Delft, The Netherlands \\ $11 \quad{ }^{5}$ Institute for Water Problems, Russian Academy of Science, Moscow, Russia
}

${ }^{\text {i }}$ Present addresses: Department of Earth Sciences, Uppsala University, Uppsala, 75236, Sweden, and Centre of Natural Hazards and Disaster Science (CNDS), Sweden

\section{Abstract}

It is crucial to be able to forecast flows and overflows in urban drainage systems to build good and effective real-time control and warning systems. Due to computational constraints, it may often be unfeasible to employ detailed 1D hydrodynamic models for real-time purposes, and surrogate models can be used instead. In rural hydrology, forecast models are usually built or calibrated using long historical time series of, for example, flow or level observations, but such series are typically not available for the ever-changing urban drainage systems. In the current study, we therefore used a fast, reservoir-based surrogate forecast model constructed from a distributed urban drainage model. Thus, we did not rely directly on historical time series data. Forecast models should preferably be able to update their internal states based on observations to ensure the best initial conditions for each forecast. We therefore used the Ensemble Kalman filter to update the surrogate model before each forecast. Water levels or flow observations were assimilated into the model either directly, or indirectly using rating curves. The model forecasts were validated against observed flows and overflows. The results showed that model updating improved the forecasts up to two hours ahead, but also that updating using water level observations resulted in better flow forecasts than assimilation based on flow data. Furthermore, updating with water level observations was insensitive to changes in the noise formulation used for the Ensemble Kalman filter, meaning that the method is suitable for operational settings where there is often little time and data for fine-tuning.

\section{Keywords}

CSO, data assimilation; Ensemble Kalman filter; flow forecasts; surrogate model; urban drainage 


\section{Introduction}

Many cities around the world are currently facing issues with changing precipitation patterns and increased urbanization, which may lead to an increased amount of runoff (Angel et al., 2011; Kaspersen et al., 2017). The sewer systems in many older cities were constructed more than 100 years ago. They are therefore not designed to cope with the increased inflow, which leads to discharges to the environment through sewer overflows or bypasses at the wastewater treatment plants. We can use real-time urban drainage models to investigate and diminish these negative effects. The models span from simple linear reservoir models to detailed 1D hydrodynamic models (high-fidelity (HiFi) models) such as MIKE URBAN, SWMM and InfoWorks.

Many utilities already have HiFi models of their systems for planning purposes. The main focus of such models is to investigate the impact of system alterations, and to inspect if the system complies with the design criterion, such as frequency of full running pipes or flooding occurrences. They can be constructed almost entirely from the utility company's asset database with information on pipe locations, diameters, etc. Sometimes, such models can even be used without any calibration and still provide satisfactory results if the asset database is well maintained and the hydrology is simple (Borup et al., 2016). When they are calibrated, this is often done as a lumped correction of the catchment properties based on a single (often downstream) in-sewer observation (see, for example, Barco et al. (2008); Rasmussen et al. (2008); and Tscheikner-Gratl et al. (2016)).

Forecast models are important in supporting urban drainage management, for example, in connection with model predictive control (MPC) of urban drainage systems (Lund et al., 2018a). HiFi models are often not applicable in real-time due to the computational demand, which is the main incentive for using simplified models; however, it is typically at the expense of loss of performance. In rural hydrology, forecast models are therefore often conceptual models derived from and/or calibrated on long time series of historical data. There are also examples of this type of models in urban drainage research (see, for example, Löwe et al. (2014); Pedersen et al. (2016); and Thorndahl et al. (2013)), but such models are usually not applied in operational settings due to a general lack of long historical time series data that can be used to build and calibrate the models. This lack of useful time-series data is partly due to the cost of maintaining sensors in the harsh environment in the sewer systems, but also because sewer systems are ever-changing. Hence, historical sensor data might not be useful for constructing and/or calibration of simpler and faster forecast models for current conditions (including both conceptual and data-driven models). The collection of new observations can be both expensive and time-consuming. Instead, a surrogate that emulates the response of an existing HiFi model can be constructed (see, for example, Carbajal et al. (2017); Thrysøe et al. (2019); and Wolfs et al. (2013)), which due to the reasonably accurate representation of the physical system components in the HiFi model may be applicable without further calibration. Compared to purely data-driven models (for example, neural networks) such emulators furthermore have the advantage of being able to 
estimate conditions outside the range previously observed by sensors in the system, and to estimate flows and levels in locations without sensors.

When there is sensor data from the urban drainage system, it is normally present as either level or flow measurements. Level sensors are cheaper, more reliable and easier to maintain than flow sensors, which makes level measurements the most common type of observation. It can be difficult, however, to build forecast models based solely on level measurements, since these do not directly relate to the flow.

Any model is only a partial representation of reality and cannot fully replicate the dynamics of the real system. Thus, models are subject to different sources of uncertainty which negatively affect their performance. In urban drainage systems, the deviations between observations and model can arise from uncertainties in the model structure (as, for example, an improper description of non-linear processes, such as backwater effects, or the exclusion of interactions between the drainage system and the surroundings through infiltration/exfiltration), uncertain parameter values, uncertain initial conditions (for example, the initial wetting of the soil affecting the amount of runoff), as well as uncertainties in measurements of precipitation, flow, level, etc. (Thorndahl et al., 2008). Data assimilation (also denoted 'model updating') can be used to prevent the model from drifting away from reality and for obtaining a better estimate of the current system state (Houser et al., 2012; Hutton et al., 2010). Data assimilation aims at improving model performances and forecasts by combining the knowledge from both the model and the observations to dynamically update model states, input, parameters and/or output (Refsgaard, 1997).

Data assimilation in rural hydrology is a relatively well-proven field of research (Liu et al., 2012). The Kalman filter (KF) is a classic data assimilation scheme, which relies on assumptions of linear models and where the input, model and observations noises are assumed to be normally distributed. Using the KF to update non-linear models can lead to sub-optimality and instability issues (Abaza et al., 2015). This has inspired the development of other variations of the filter, such as the Extended Kalman filter and the Ensemble Kalman filter (EnKF), where the EnKF furthermore has the advantage of being computational efficient for large models (Evensen, 1994).

For a filter to be relevant for urban drainage modelling, it needs to be able to handle nonlinearities, as sewer systems have a highly non-linear behaviour with both backwater effects and internal and external overflows. Literature on data assimilation in urban drainage models is, contrary to rural hydrology, relatively limited. Borup et al. (2014) hypothesize that this is because ambitions with respect to real-time control and flow forecasting have historically been low, and that computational power and data acquisition technology, which makes it possible to apply real-time updating, has only developed sufficiently in recent years. The reported data assimilation techniques applied within urban drainage span from simple methods, where uncertainty is disregarded, to quite sophisticated methods. For example, Hansen et al. (2014) applied simple direct insertion to correct the water level in a HiFi model. Here, the states were directly changed to the value of the observation, and 
uncertainty estimations were thus not required. Direct insertion may lead to model shocks that cause the simulated outflow at the updated location to oscillate. Furthermore, it can only be used to propagate the information to downstream parts (Hansen et al., 2014; Houser et al., 2012). Borup et al. (2011) indirectly updated the flows in a HiFi model by employing a dampened feedback from downstream model residuals to the hydrological states of the model, while Hutton et al. (2014) updated the states of a simplified model based on flow measurements, using a deterministic Kalman filter-type update. Both Borup et al. (2011) and Hutton et al. (2014) took the time lag of the system into account. Branisavljevic et al. (2014) applied the Extended Kalman filter to update the states in a simplified model based on water level and flow observations, and further found that subsequent bias reduction can improve the results. Borup et al. $(2014,2018)$ showed that the EnKF can be used to update the water levels throughout a HiFi model based on flow as well as water level observations. Even though the EnKF is much cheaper to run for large models than a traditional KF, the propagation of the ensemble forward in time is still computationally expensive for large HiFi models. Borup et al. (2014) used an ensemble size of 10; however, the extent of the study was limited and they did not produce flow forecasts. The presented examples demonstrate the applicability of data assimilation to urban drainage models, and at the same time show that this area allows for (and requires) much more research.

This study proposes a methodology for forecasting both flows and overflows using a distributed conceptual piecewise-linear reservoir model as surrogate for a HiFi model, without relying on long historical time series for constructing or calibrating the model. While the surrogate model is constructed purely from the parameters and results from the HiFi model, the surrogate model states are updated every time a new water level or flow observation is available using the EnKF as data assimilation method. The updated states are used as initial conditions for forecasting with the surrogate model. Since level gauges are the preferred sensors in urban drainage systems, we investigate the impact of using flow and level data, respectively, for the updating. The observations are both used directly in the data assimilation scheme, or converted via rating curves. The focus on observation type and independence of long historical time series has, to our knowledge, not been the focus of previous data assimilation research within urban drainage. A small sub-catchment in

136 Copenhagen, Denmark is used as case study. The case area has a downstream CSO structure, and the 137 surrogate model is used to make forecasts of the throttle and CSO flow from this structure. The 138 surrogate model and data assimilation scheme are set up only using data from the HiFi model and the 139 technical drawings of the inspected CSO structure to develop a method that would not need long historical time series. 


\section{Methodology}

142

143

144

145

146

147

148

149

150

151

152

153

154

155

156

157

158

159

160

161

162

163

164

165

166

167

168

169

170

171

172

173

174

\subsection{Surrogate model}

The surrogate model used in the current study represents the drainage system as a system of connected virtual tanks. Each virtual tank represents a volume of water in a specific part of the pipe system, hereafter referred to as a 'compartment'. The outflows from these compartments are calculated using a series of tabulated storage-discharge relationships, which are the main parameters of the surrogate model. These are extracted directly from the HiFi model by propagating a slowly increasing design rainfall through the model. During the surrogate model simulation, the flow at any given point in time is found by interpolating between the nearest tabulated storage-discharge values for the relevant storage volume. Each compartment can have multiple outlets, as can be seen in Figure 1a, for example, for the downstream compartment, which has both an outlet through 'CSO' and 'out downstream'. A more detailed description of the surrogate model and of its calibration can be found in Borup et al. (2017) and Thrysøe et al. (2019). The HiFi model used for estimating the surrogate model is a MIKE URBAN model (DHI, 2016), which has the desired property of being able to produce volume time series for the selected areas of the pipe network. This makes it possible to almost automatically produce the storage-discharge data used as parameters for the surrogate model. The surrogate model can be used for flow forecasting but, due to its computational speed, may also be used in a real-time control setting, including both rule-based control and MPC.

For the current study, the parameters of the surface runoff compartments are calculated from the parameters of the HiFi surface model. The surface runoff in the HiFi model is produced by multiplying the impervious area with the rainfall intensity after subtracting a catchment-specific initial loss. It is routed to the network model using a simple time-area model based on the time of concentration, $\mathrm{T}_{\mathrm{c}}$. The surface runoff in the surrogate model is also based on the total impervious area, which is easily extracted from the HiFi model's parameter file, and the rainfall intensity - but without considering the initial loss. Instead of using a time-area model for routing the runoff to the network compartments, a single linear reservoir is used where the time constant corresponds to the mean retention time when using the time-area model, which is $0.5 \mathrm{~T}_{\mathrm{c}}$.

\subsection{Ensemble Kalman filter}

In this paper we use the EnKF, which is Monte Carlo implementation of the KF where the uncertainties are represented using an ensemble of models. The EnKF allows for non-linear models and non-Gaussian uncertainty, and is less computationally demanding than the KF for large models (Evensen, 2003).

In the EnKF, an ensemble of model instances, representing the probability distribution of the model states, is propagated forward in time.

$$
\mathbf{x}_{t+1}^{\mathrm{f}, i}=M\left(\mathbf{x}_{t}^{\mathrm{a}, i}, \mathbf{u}_{t}^{i}, \mathbf{w}_{t}^{i}\right)
$$


In Eq. $1, \mathbf{x}_{t}^{\mathrm{a}, i}$ is a vector with the updated states from the previous time step for ensemble member $i$. These states are propagated forward in time by the model $M$, applying the input $\mathbf{u}_{t}^{i}$ (for example, the measured rainfall intensities) and the system noise, $\mathbf{w}_{t}^{i}$, which includes input and model uncertainty. Hereby, the vector with the forecasted states at time $t+1, \mathbf{x}_{t+1}^{\mathrm{f}, i}$, is obtained. The states of the $N$ individual ensemble members form the model state matrix (Eq. 2).

$$
\mathbf{X}_{t+1}^{\mathrm{f}}=\left[\mathbf{x}_{t+1}^{\mathrm{f}, 1}, \mathbf{x}_{t+1}^{\mathrm{f}, 2}, \ldots, \mathbf{x}_{t+1}^{\mathrm{f}, N}\right]
$$

The error covariance, $\mathbf{P}_{t}^{\mathrm{f}}$, is estimated from the ensemble (Eqs. 3-5)

$$
\begin{gathered}
\overline{\mathbf{x}}_{t+1}^{\mathrm{f}}=\frac{1}{N} \sum_{i=1}^{N} \mathbf{x}_{t+1}^{\mathrm{f}, i} \\
\mathbf{A}_{t+1}^{\mathrm{f}}=\left[\mathbf{x}_{t+1}^{\mathrm{f}, 1}-\overline{\mathbf{x}}_{t+1}^{\mathrm{f}}, \mathbf{x}_{t+1}^{\mathrm{f}, 2}-\overline{\mathbf{x}}_{t+1}^{\mathrm{f}}, \ldots, \mathbf{x}_{t+1}^{\mathrm{f}, N}-\overline{\mathbf{x}}_{t+1}^{\mathrm{f}}\right] \\
\mathbf{P}_{t+1}^{\mathrm{f}}=\frac{1}{N-1} \mathbf{A}_{t+1}^{\mathrm{f}} \mathbf{A}_{t+1}^{\mathrm{fT}}
\end{gathered}
$$

Whenever observations, $\mathbf{z}$, become available, the state ensemble is updated by

$$
\mathbf{x}^{\mathrm{a}, i}=\mathbf{x}^{\mathrm{f}, i}+\mathbf{K}\left(\mathbf{z}^{i}-H\left(\mathbf{x}^{\mathrm{f}, i}\right)\right), \quad \mathbf{z}^{i}=\mathbf{z}^{+} \mathbf{v}^{i}
$$

For simplicity, the time index, $t$, is omitted in Eq. 6 and in the subsequent equations In Eq. $6, \mathbf{z}^{i}$ is the $i^{\text {th }}$ perturbed observation and $\mathbf{v}^{i}$ is the $i^{\text {th }}$ observation noise, which is assumed to have zero mean and covariance matrix $\mathbf{R}$. If observations are uncorrelated, the observation error covariance will become a diagonal matrix. $H$ is a function relating model states to the observations. In the standard KF implementation, $\mathbf{H}$ is a matrix and thus assumes a linear relation between observations and states. The EnKF allows non-linear relations between states and observations, and is therefore written instead as a vector function $H$. The term $\mathbf{z}^{i}-H\left(\mathbf{x}^{\mathrm{f}, i}\right)$ is the deviation between the observations and the modelled equivalents of the observations. $\mathbf{K}$ is the Kalman gain matrix, which weights the trust in the model and observations. A Kalman gain of 0 reflects perfect trust in the model and, in this case, no update will take place. On the other hand, a Kalman gain of 1 will update the model such that it would exactly correspond to the measured value. Neither of these options is realistic, as both the model and the observations are uncertain. The Kalman gain is calculated as

$$
\mathbf{K}=\mathbf{P}^{\mathrm{f}} \mathbf{H}^{\mathrm{T}}\left(\mathbf{H} \mathbf{P}^{\mathrm{f}} \mathbf{H}^{\mathrm{T}}+\mathbf{R}\right)^{-1}
$$

In the EnKF, $\mathbf{P}^{\mathrm{f}} \mathbf{H}^{\mathrm{T}}$ is approximated by the ensemble covariance between the model states and the modelled observations (Eq. 8), while $\mathbf{H P}^{\mathrm{f}} \mathbf{H}^{\mathrm{T}}$ can be approximated by the ensemble variance of the modelled observations (Eq. 9). 


$$
\begin{gathered}
\mathbf{P}^{\mathrm{f}} \mathbf{H}^{\mathrm{T}}=\frac{1}{N-1} \sum_{i=1}^{N}\left[\left(\mathbf{x}^{\mathrm{f}, i}-\overline{\mathbf{x}}^{\mathrm{f}}\right)\left(H\left(\mathbf{x}^{\mathrm{f}, i}\right)-{\overline{H\left(\mathbf{x}^{\mathrm{f}, i}\right)}}^{\mathrm{T}}\right]\right. \\
\mathbf{H P}^{\mathrm{f}} \mathbf{H}^{\mathrm{T}}=\frac{1}{N-1} \sum_{i=1}^{N}\left[\left(H\left(\mathbf{x}^{\mathrm{f}, i}\right)-\overline{H\left(\mathbf{x}^{\mathrm{f}, i}\right)}\right)-\left(H\left(\mathbf{x}^{\mathrm{f}, i}\right)-\overline{H\left(\mathbf{x}^{\mathrm{f}, i}\right)}\right)^{\mathrm{T}}\right]
\end{gathered}
$$

197

where

$$
\overline{H\left(\mathbf{x}^{\mathrm{f}, i}\right)}=\frac{1}{N} \sum_{i=1}^{N} H\left(\mathbf{x}^{f, i}\right)
$$

198

The EnKF is one of the most frequently used techniques for data assimilation in hydrological applications. The advantages of using the EnKF are: 1) the possibility of using non-linear models (Clark et al., 2008; Liu et al., 2012; McMillan et al., 2013); 2) the model does not have to be formulated in state-space as in some of the other updating schemes (Clark et al., 2008); 3) it is computationally efficient for large models compared to the covariance propagation in the standard KF (Clark et al., 2008; Rakovec et al., 2012); 4) it is robust (Liu et al., 2012); and 5) it fits well into the probabilistic approach related to forecasting schemes (Clark et al., 2008). Even though non-linear models can be applied, the model update itself is linear, which means that the filter is optimal only for strictly linear systems with Gaussian errors. This rarely holds in hydrological models, but the filter can still be successfully applied despite not being optimal (Clark et al., 2008; McMillan et al., 2013).

\section{Experimental setup}

\subsection{Case area}

The case area is a 1.48-km² urban catchment located in the Damhusåen catchment in the western part of the Greater Copenhagen area, Denmark. This area is also subject to an ongoing study on model predictive control (Lund et al., 2017). The outflow from the downstream CSO structure is influenced by backwater effects at high water levels. Under normal conditions the flow runs through the CSO structure and on to the main collector through a throttle pipe ('out downstream', see Figure 1), but during heavy rainfall the capacity of the throttle pipe is superseded, resulting in water backing up into the pipe system. Once the weir level is exceeded in the CSO structure, it eventually causes overflow into the local creek. A simplified schematic of the CSO structure is shown in Figure 1c. A minor fraction of the upstream flow runs to the sewer system in the neighbouring catchment (through 'out west', see Figure 1) but otherwise there are no direct interactions with the drainage systems in the surrounding areas.

The case area is one of many comparable catchments from which the sewage can be led to a newly constructed 3.2-km tunnel via centralized control schemes that aim at reducing overflows from 
223 the system as well as ensuring optimal operation of the downstream wastewater treatment plant. When

224 there is CSO from one of the sub-catchments connected to the tunnel, the water enters the channelized

225 local creek from which it quickly ends up close to a planned bathing area at the coast. This means that

226 a CSO from this catchment can influence the objective of the control of the larger system. It is

227 therefore important to be able to model and forecast both flows and CSOs from these catchments.

\section{$228 \quad 3.2$ Models}

229 The MIKE URBAN model used as the HiFi model has been provided by the utility company 230 (HOFOR), and the surrogate model has been based on this model. We have subsequently reduced the 231 extent of the HiFi model to include only the part important for the investigated CSO structure. This 232 part consists of the area upstream of the CSO structure as well as a smaller downstream part, which is 233 important in accounting for the backwater effects into the CSO structure (Figure 1a). The reduced 234 HiFi model contains 233 nodes, 97 links, 67 sub-catchments and the CSO structure, and will from 235 here on simply be denoted 'the HiFi model'.

236 The surrogate model representing the network part of the HiFi model consists of five 237 compartments of comparable spatial extent (Figure 1). The surrogate model does not cover the full 238 extent of the HiFi model because the surrogate model cannot take backwater effects into account, 239 which is why it is not needed to model the flow downstream of the CSO structure. Each network 240 compartment receives surface inflow from a single runoff compartment (Figure 1b). Figure 2 shows 241 the piecewise-linear storage-discharge curves for each of the compartments. The surrogate model is 242 run with 60-second time steps. This study has shown that the surrogate model is 3500 times faster 243 than the HiFi model when the latter is run with adaptive time steps between 10 and 60 seconds. This 244 speed of the surrogate model is a huge benefit when using the model in combination with EnKF.

245 The exclusion of dry weather flow (DWF) can lead to non-optimal updating of the model, and a 246 DWF component is therefore added to the downstream flow. This component is constructed using a double sine curve and calibrated on the DWF pattern in the HiFi model. 


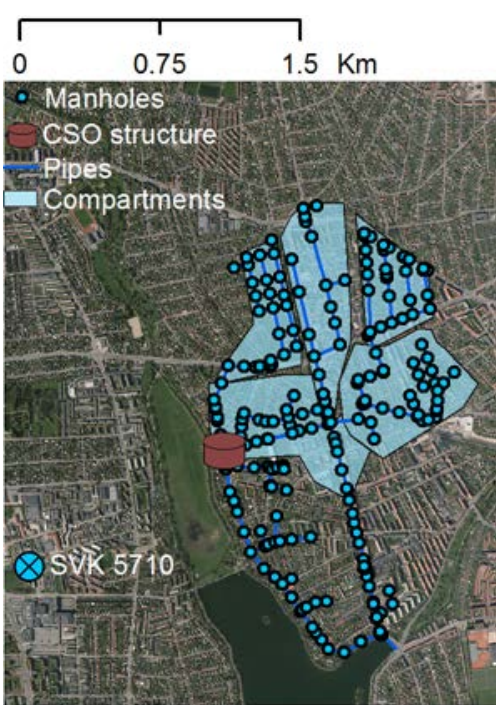

(a)

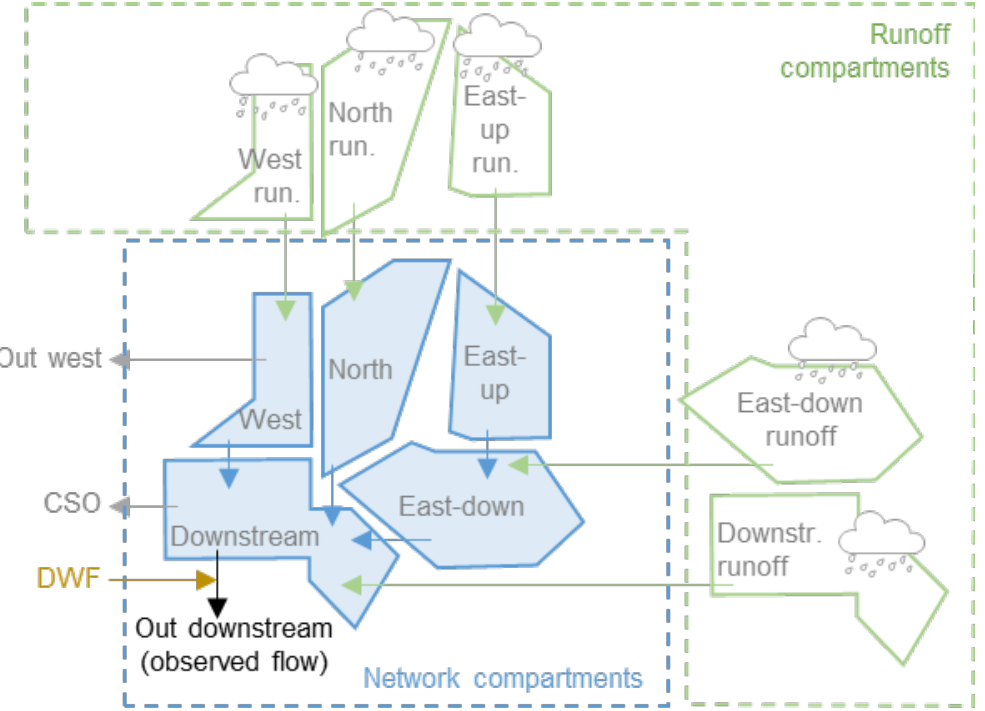

(b)

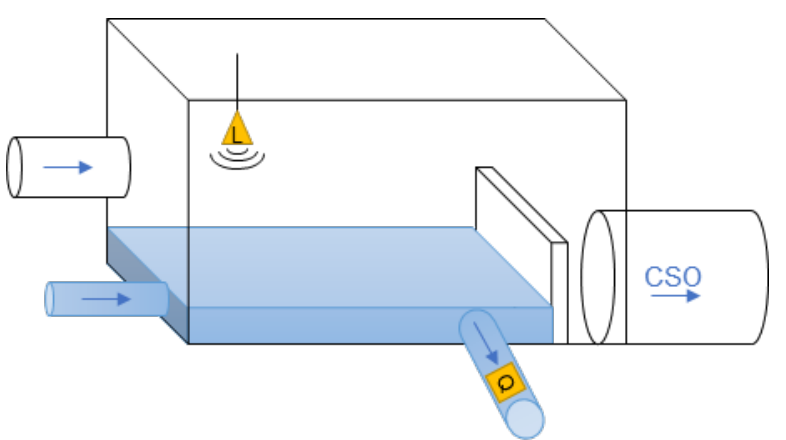

(c)

Figure 1. (a) HiFi model, the approximate division of pipes into compartments and rain gauge (SVK 5710); (b) conceptual drawing of the compartment model; (c) Simplified schematic of the CSO structure - the two yellow shapes mark the level measurement $(\mathrm{L})$ inside the CSO structure and the flow measurement $(\mathrm{Q})$ in the throttle outlet from the CSO structure. 


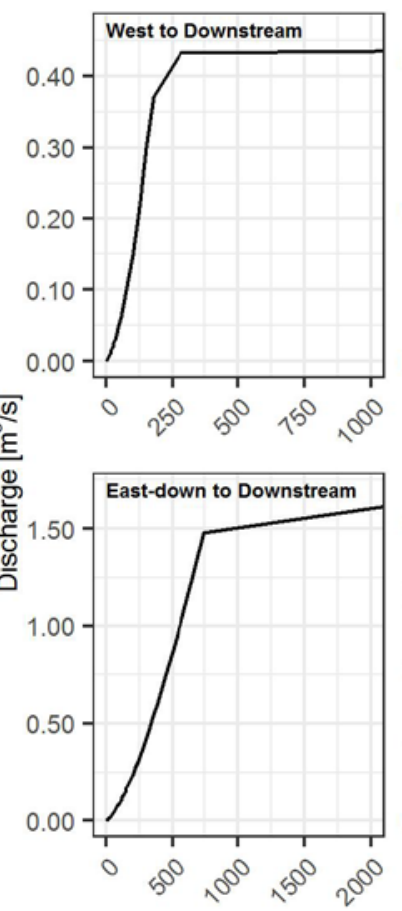

254

255

256

257

258

259

260

261

262

263

264

265

266

267

268

269

270

\subsection{Input and observations}
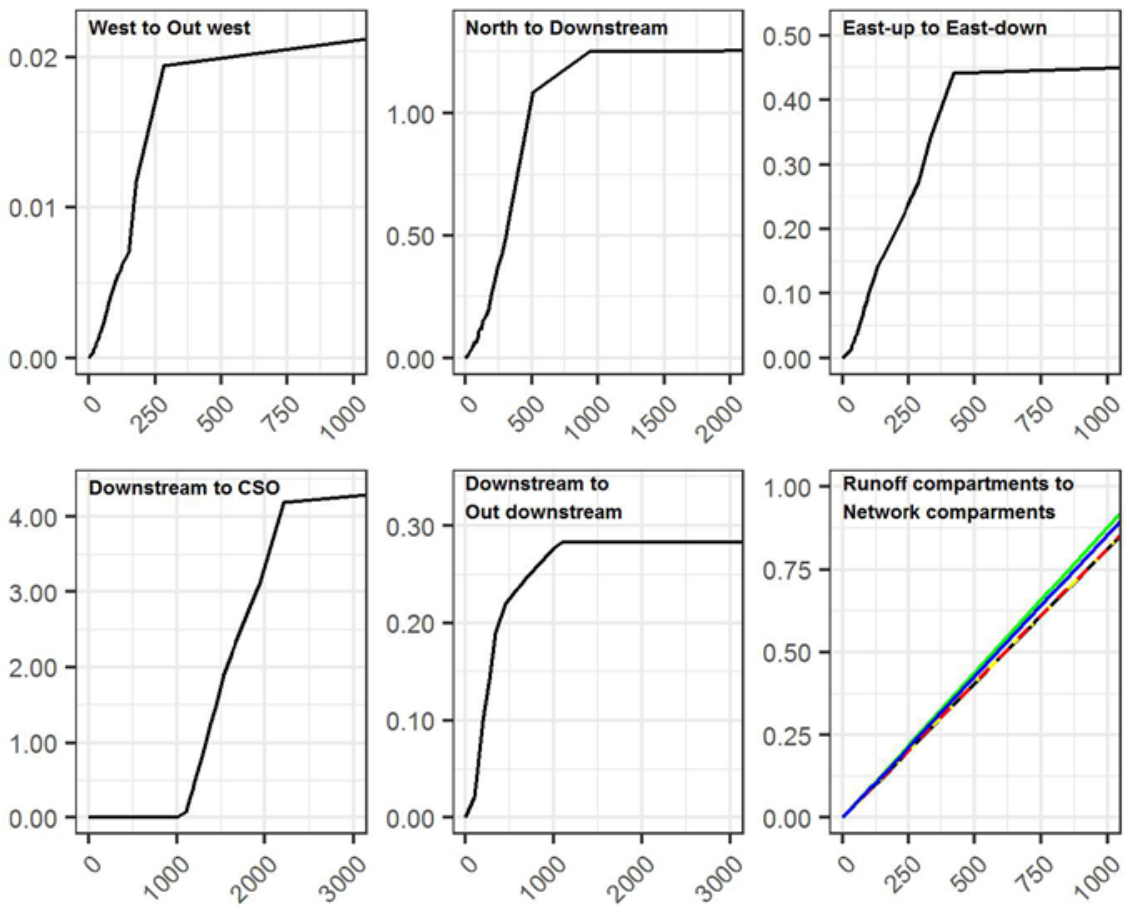

Volume $\left[\mathrm{m}^{3}\right]$

Figure 2. Storage-discharge curves for all compartments. The curves for the runoff compartments have been plotted with different colours. All curves have been cut at a volume that allows for better visualization of the dynamics.

Time displacement caused by the time it takes for a rain cell to travel from the rain gauge to the catchment can severely reduce the quality of the forecast from updated models (Borup et al., 2013). In this study, rainfall observations come from the rain gauge SVK 5710 (Jørgensen et al., 1998) placed about $1.2 \mathrm{~km}$ south-west of the considered sub-catchment. This is sufficiently close to the case area to expect no large impacts of the time displacement. The flow data (Q) comes from an electromagnetic sensor placed inside the throttle pipe leading out from the CSO structure, while the level sensor (L) is an ultrasonic sensor that directly observes the water level in the CSO chamber, Figure 1c. All observations have a frequency of 1 minute.

Figure 3a shows the relationship between the measured flow and level data for seven rain events from August 1 to October 30, 2016. There is a clear, though not unambiguous, relationship. Figure 3b shows the level and flow modelled by the HiFi model for the same seven rain events. It is clear that the variability in the measurements is larger in reality than in the model, and that the bottom levels and crest levels are different; however, the overall shape fits well. 


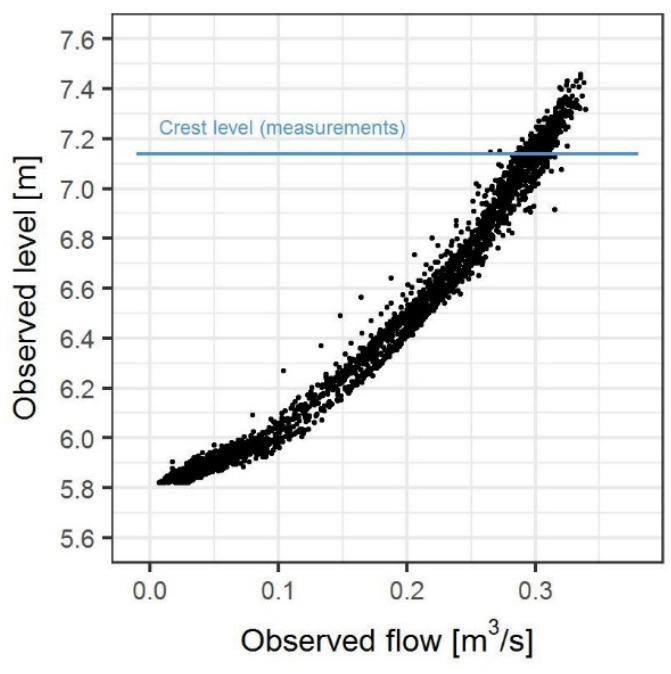

(a)

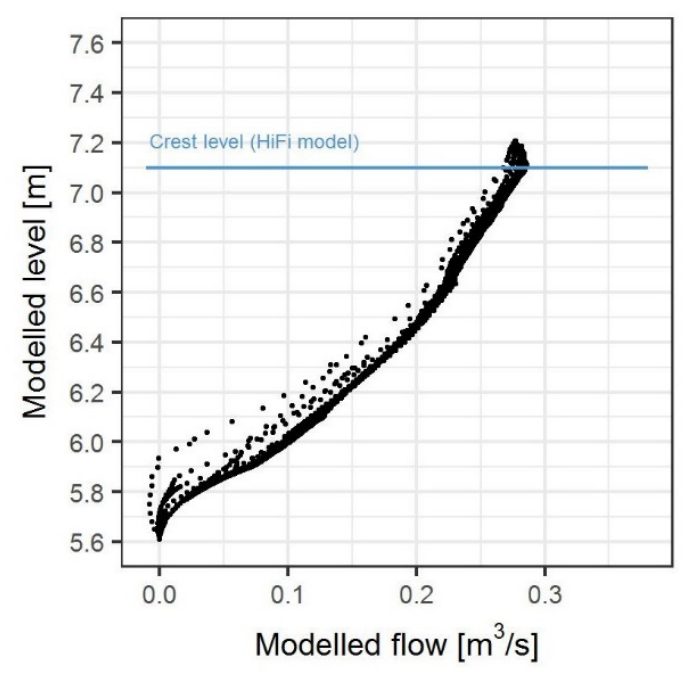

(b)

Figure 3. Relationship between flow and levels in (a) measurements, and (b) HiFi model.

\section{3}

\subsection{Observations used for updating}

The data assimilation is carried out for a period of approximately one month from June 16 to July 12, 2016, during which several overflows (water level above crest level) occurred, and during which the data was visually assessed as being trustworthy, see Figure 4. Missing and erroneous data in the flow and level measurements have been replaced with the last occurring flow and level, respectively.

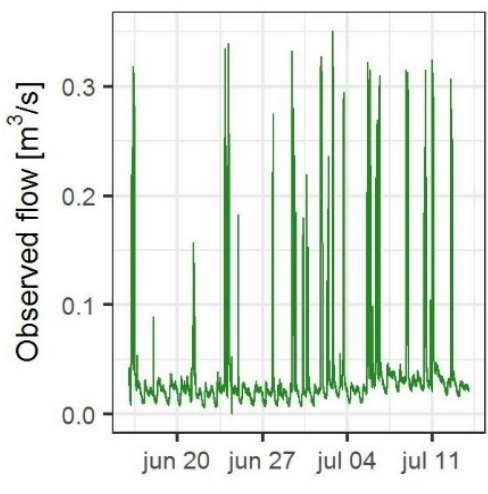

(a)

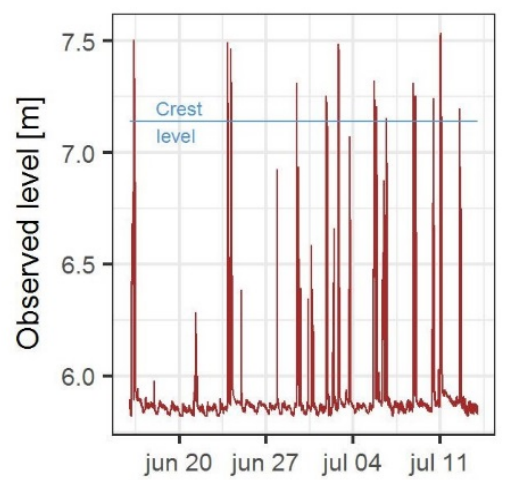

(b)

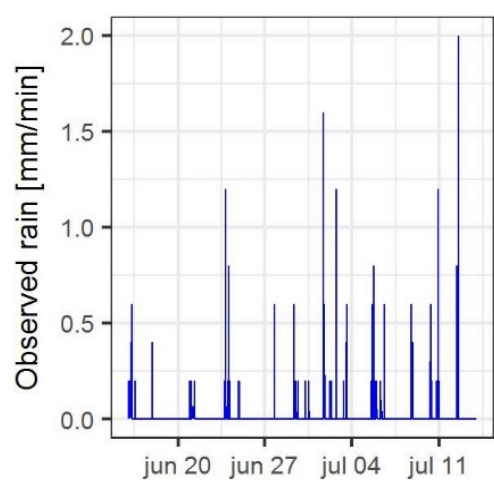

(c)

Figure 4. Observations in the data assimilation period: (a) flow; (b) level; (c) rain.

Both the flow and level observations are used for updating in this study. The compartment model uses volume as the internal state and describes the flow out of compartments, meaning that the model can be updated either with the flow out of the downstream compartment or via the volume in the same compartment. We examine four experimental setups related to the handling of the observation in the EnKF scheme:

1) EnKF-Q Updating with the flow measurement directly.

2) EnKF-QV Converting the flow measurement to volume, and using this volume for updating.

3) EnKF-LV Converting the level measurement to volume, and using this volume for updating.

4) EnKF-LQ Converting the level measurement to flow, and using this flow for updating. 
A conversion between the measured quantity and the quantity used for updating is done using rating curves for the experimental setups EnKF-QV, EnKF-LV and EnKF-LQ. These curves are created using the HiFi model, which is run from August 1 to October 30 (outside the time period later used for data assimilation). We are only interested in rainy situations and therefore choose seven rain events in this period (also used to create Figure 3). The levels of the CSO structure in the HiFi model differ from the actual levels of the CSO structure on the technical drawing (the level of the bottom is $21 \mathrm{~cm}$ lower in the HiFi model than on the drawing and the crest level is situated $4 \mathrm{~cm}$ lower), as seen in Figure 3. We use this information to correct the levels in the HiFi model before establishing the two level-dependent rating curves (Figure 5). In Figure 5, the grey circles indicate the results from the HiFi model (after correction of the levels). The quantity being converted (x axis) is divided into 14 equally large bins and a straight line is fitted to the data within each bin. Subsequently, the centre of each line (one for each bin, the red circles) are connected with the minimum and maximum values, creating 15 regression line segments that together constitute the rating curve (red line).
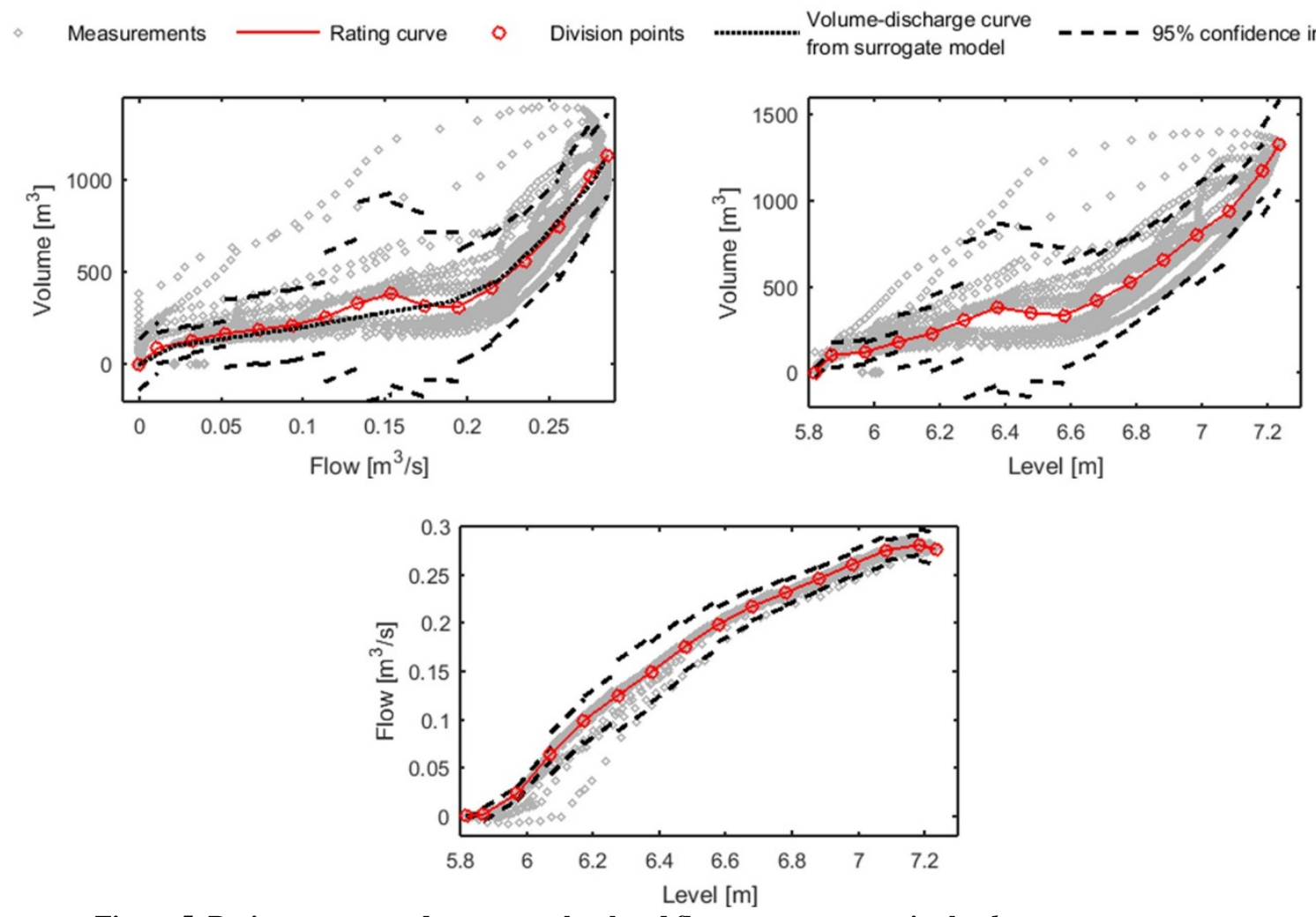

Figure 5. Rating curves used to convert level and flow measurements in the downstream compartment.

In KF, the measurement operator, $H$, would normally be used to map between states and observations. We choose to do the conversion outside the filter implementation in order to use the rating curves to estimate the observation error. Thus, for EnKF-Q and EnKF-LQ, the measurement operator, $H$, which converts the state to the modelled measurement, is the piecewise-linear compartment model that converts the downstream network compartment state to the throttle flow out of the CSO structure. For EnKF-QV and EnKF-LV, the measurement operator, $H$, is a vector of zeros 
310 with a single value of one that picks the volume for the downstream compartment from the state 311 vector.

\section{$312 \quad 3.5 \quad$ Implementation of the Ensemble Kalman filter}

313 The data assimilation is performed in MATLAB by connecting the surrogate model with the EnKF-

314 MATLAB toolbox developed by Sakov (2013). The state vector for the updating consists solely of the

315 volumes in the five network compartments, since a preliminary study showed that additional updating 316 of the volume in the runoff compartments did not improve the results (Lund et al., 2018b).

317 Choosing the ensemble size is a trade-off between the performance of the updating and the 318 computational cost. We performed initial tests with ensemble sizes of 10, 20, 50, 100, 200 and 500 319 members. Using 10 and 20 members provided poor results while 50-100 members and above provided 320 similar results in terms of the squared error on forecast flows. To avoid negative impacts on the 321 performance due to an insufficient ensemble size, we choose an ensemble size of 100 . This is still feasible in terms of computational cost due to the efficiency of the surrogate model.

In EnKF applications, all uncertainties have to be represented as realizations of random noise. This means that model forcing, observations and state variables will be perturbed according to the specified noise properties. The specification of these can have a big impact on the filter performance (Zhang et al., 2015). When long historical observations are available, the various noises can to some extent be estimated or optimized from the data, but in the current setup we constrain ourselves either to derive the noise terms from the HiFi model or to simply estimate a range of reasonable noise terms. We assume that the uncertainty mainly originates from the rainfall observations as well as the runoff and network parts of the model. There is also uncertainty related to the observations. Therefore, we perturb the rainfall input, the model compartment states and the observations. The noise formulation for the observations differs, depending on the experimental setup. Table 1 gives an overview of the choices made for the noise formulations, while the subsequent sections describe each of the three error sources in detail.

Table 1: Noise formulations for rainfall, states and observations.

\begin{tabular}{|c|c|c|c|}
\hline & Perturbation & & \\
\hline Rainfall & $\mathbf{I}_{\mathrm{ens}}(t)=\mathrm{I}_{\mathrm{obs}}(t) \cdot \varepsilon_{\mathrm{I}}(\Delta \mathrm{t})$ & $\varepsilon_{\mathrm{I}}(\Delta \mathrm{t}) \sim \mathrm{U}[0.5,1.5]$ & $\Delta \mathrm{t} \sim \mathrm{U}[1,10]$ \\
\hline States & $\mathbf{V}_{\text {ens }}(t)=\mathrm{V}_{\bmod }(t) \cdot \varepsilon_{\mathrm{V}}(t)$ & $\varepsilon_{\mathrm{V}}(t) \sim \mathrm{N}\left(1,\left(\alpha_{\mathrm{V}} \mathrm{V}_{\mathrm{mod}}\right)^{2}\right)$ & $\begin{array}{l}\alpha_{\mathrm{V}}=\left[\alpha_{\mathrm{V}, \text { netw. }}, \alpha_{\mathrm{V}, \text { runoff }}\right]=[0.05,0.1] ; \\
\mathrm{V}_{\mathrm{mod}}=\text { modelled volume }\end{array}$ \\
\hline \multicolumn{4}{|c|}{ Observations in each experimental setup } \\
\hline - EnKF-Q & $\mathbf{Q}_{\mathrm{obs}}(t)=\mathrm{Q}_{\mathrm{obs}}(t) \cdot \varepsilon_{\mathrm{Q}}(t)$ & $\varepsilon_{\mathrm{Q}}(t) \sim \mathrm{N}\left(1,\left(\alpha_{\mathrm{Q}} \mathrm{Q}_{\mathrm{obs}}\right)^{2}\right)$ & $\alpha_{Q}=0.1 ; Q_{o b s}=$ observed flow \\
\hline - EnKF-QV & $\mathbf{V}_{\mathrm{QV}}(t)=\mathrm{V}_{\mathrm{QV}}(t)+\varepsilon_{\mathrm{QV}}(t)$ & $\varepsilon_{\mathrm{QV}}(t) \sim \mathrm{N}\left(0,\left(\beta \sigma_{\mathrm{V}}\right)^{2}\right)$ & $\beta=1 ; \sigma_{\mathrm{V}}=$ from HiFi model results \\
\hline - EnKF-LV & $\mathbf{V}_{\mathrm{LV}}(t)=\mathrm{V}_{\mathrm{LV}}(t)+\varepsilon_{\mathrm{LV}}(t)$ & $\varepsilon_{\mathrm{LV}}(t) \sim \mathrm{N}\left(0,\left(\beta \sigma_{\mathrm{V}}\right)^{2}\right)$ & $\beta=1 ; \sigma_{\mathrm{V}}=$ from HiFi model results \\
\hline - EnKF-LQ & $\mathbf{Q}_{\mathrm{LQ}}(t)=\mathrm{Q}_{\mathrm{LQ}}(t)+\varepsilon_{\mathrm{LQ}}(t)$ & $\varepsilon_{\mathrm{LQ}}(t) \sim \mathrm{N}\left(0,\left(\beta \sigma_{\mathrm{Q}}\right)^{2}\right)$ & $\beta=1 ; \sigma_{\mathrm{Q}}=$ from HiFi model results \\
\hline
\end{tabular}




\subsubsection{Perturbation of rainfall data}

The measured rainfall is perturbed by multiplying it with a random factor $\varepsilon_{\mathrm{I}}$ that is drawn from a uniform distribution between 0.5 and 1.5 every $\Delta t$ minutes, where $\Delta t$ itself is a random variable that is drawn from a uniform distribution between 1 and 10 minutes at the same time as $\varepsilon_{\mathrm{I}}$. Hereby, we obtain the rainfall ensemble, $\mathbf{I}_{\text {ens. }}$ The limits of the uniform distribution are chosen based on a sensitivity study carried out by Lund et al. (2018b).

$$
\mathbf{I}_{\text {ens }}(t)=\mathrm{I}_{\text {obs }}(t) \cdot \varepsilon_{\mathrm{I}}(\Delta \mathrm{t}), \quad \varepsilon_{\mathrm{I}}(\Delta \mathrm{t}) \sim \mathrm{U}[0.5,1.5], \Delta \mathrm{t} \sim \mathrm{U}[1,10]
$$

Temporal correlation in the error is important, as rapid fluctuations around the mean can otherwise cancel themselves out as the rainfall errors are aggregated in the model, which can lead to insufficient ensemble spread. The temporal persistence in the rainfall observation error can be explained by the nature of a tipping bucket rain gauge that integrates the rain since the last tip, and by the fact that rainfall observations are affected by sudden changes in wind direction, etc. This way of making ensemble representation of the rainfall error has previously been used in an urban runoff data assimilation study (Borup et al., 2014). The method makes it possible to obtain a larger spread in the resulting runoff ensemble without having negative factors or affecting the ensemble mean, compared to using, for example, a Gaussian random walk, which can be a desirable property in data assimilation studies.

\subsubsection{Perturbation of states}

We perturb the states (volumes) of the compartments as

$$
\mathbf{V}_{\text {ens }}(t)=\mathrm{V}_{\text {mod }}(t) \cdot \varepsilon_{\mathrm{V}}(t), \quad \varepsilon_{\mathrm{V}}(t) \sim \mathrm{N}\left(1,\left(\alpha_{\mathrm{V}} \mathrm{V}_{\text {mod }}\right)^{2}\right)
$$

where $\mathrm{V}_{\text {mod }}$ is the modelled volume in the runoff compartment at time $t$. The proportional noise term, $\varepsilon_{\mathrm{V}}$, is normally distributed with a standard deviation of $\alpha_{\mathrm{V}} \mathrm{V}_{\text {mod }}$, with $\alpha_{\mathrm{V}}=\left[\alpha_{\mathrm{V}, \text { netw. }}, \alpha_{\mathrm{V}, \text { runoff }}\right]$. In this study, values of $\alpha_{V \text {,netw. }}$ for network compartments and $\alpha_{V \text {,runoff }}$ for runoff compartments equal 0.05 and 0.1, respectively. These are used as default estimates of the state noise. Values of 0 and 0.2 are also applied (only noise on runoff compartments) to explore how sensitive the model is to the state perturbation.

To make the updating more robust and limit the dispersion of the ensemble within a reasonable range, we use the upper volume limit of the storage-discharge curves as the maximum possible compartment state value. If an ensemble member has a volume larger than this value, its volume is set to be equal to the maximum value. This can potentially lead to an ensemble collapse if all the ensemble members are set to the maximum value; however, this potential problem is mitigated by the fact that the ensemble will be dispersed again when water flows out of the compartment and new noisy input is added at the next time step. 
For EnKF-Q, we perturb the measured flow, $\mathrm{Q}_{\mathrm{obs}}$, by assuming a normally distributed, proportional error, $\varepsilon_{\mathrm{Q}}$, with a standard deviation of $\alpha_{\mathrm{Q}} \mathrm{Q}_{\mathrm{obs}}$. The standard deviation is proportional to the flow, meaning that the larger flow will also have the larger error. In this paper, $\alpha$ is set to 0.1 (default) based on Bertrand-Krajewski et al. (2003). However, values of 0.05 and 0.2 are also tested to assess the sensitivity of this parameter.

$$
\mathbf{Q}_{\mathrm{obs}}(\mathrm{t})=\mathrm{Q}_{\mathrm{obs}}(\mathrm{t}) \cdot \varepsilon_{\mathrm{Q}}(\mathrm{t}), \quad \varepsilon_{\mathrm{Q}}(\mathrm{t}) \sim \mathrm{N}\left(1,\left(\alpha_{\mathrm{Q}} \mathrm{Q}_{\mathrm{obs}}\right)^{2}\right)
$$

374

375

376

377

378

379

380

381

382

383

384

385

For EnKF-QV, EnKF-LV and EnKF-LQ, there are errors arising both from the measurement itself and from the conversion via the rating curve. We assume that the error in the measurement per se is much smaller than the one occurring from using the rating curve and therefore we are neglecting the measuring error. The data from the HiFi model simulation shown in Figure 5 is used to establish the observation noise description for the EnKF. The standard deviation is determined for the data around the rating curve for each of the 15 line segments (see the 95\% confidence level in Figure 5 - dashed black line). Proportional noise terms, as used in EnKF-Q, are applied to indicate that the noise becomes larger for higher observed values. The change in noise depending on the measured value is, however, already embedded in the noise formulation obtained from the HiFi model (visible as narrow or broad confidence intervals in Figure 5); thus, we use additive noise terms in EnKF-QV, EnKF-LV and EnKF-LQ:

$$
\begin{array}{cc}
\mathbf{V}_{\mathrm{QV}}(\mathrm{t})=\mathrm{V}_{\mathrm{QV}}(\mathrm{t})+\varepsilon_{\mathrm{QV}}(\mathrm{t}), & \varepsilon_{\mathrm{QV}}(\mathrm{t}) \sim \mathrm{N}\left(0,\left(\beta \sigma_{\mathrm{V}}\right)^{2}\right) \\
\mathbf{V}_{\mathrm{LV}}(\mathrm{t})=\mathrm{V}_{\mathrm{LV}}(\mathrm{t})+\varepsilon_{\mathrm{LV}}(\mathrm{t}), & \varepsilon_{\mathrm{LV}}(\mathrm{t}) \sim \mathrm{N}\left(0,\left(\beta \sigma_{\mathrm{V}}\right)^{2}\right) \\
\mathbf{Q}_{\mathrm{LQ}}(\mathrm{t})=\mathrm{Q}_{\mathrm{LQ}}(\mathrm{t})+\varepsilon_{\mathrm{LQ}}(\mathrm{t}), & \varepsilon_{\mathrm{LQ}}(\mathrm{t}) \sim \mathrm{N}\left(0,\left(\beta \sigma_{\mathrm{Q}}\right)^{2}\right)
\end{array}
$$

When using the noise estimation directly from the HiFi model, $\beta$ is set to 1 (referred to as default settings). In order to explore the sensitivity of the measurement error, we scale the standard deviation by changing $\beta$ to $0.001,0.01$ and 0.1 . Values of 10 and 100 have also been applied but these gave extremely poor results, since the standard deviations extracted from Figure 5 are already quite large, especially for the conversions into volumes. A larger standard deviation may therefore result in negative volumes, which the model converts to zeros, thus resulting in a biased ensemble. These results are therefore not shown.

\subsection{Evaluation measures}

The objective of the study is to establish whether, and by how much, we can improve the flow and overflow forecast performance of a surrogate model by using EnKF. We use flow forecasts up to 180 minutes into the future to quantify the performance, both to give an indication of the quality of the update of the internal states and to examine the potential of using data assimilation in a forecast 
setting. In this study, we assume that the forecast rainfall equals the measured one. This, however, still includes some uncertainty due to representation error of the rain gauge. We apply deterministic forecasts, meaning that we use the mean of the updated states as initial conditions for a single forecast model run. We make deterministic forecasts because this is what many model-based control schemes, such as MPC, can currently manage. The four experimental setups are compared with the surrogate model baseline (the surrogate model without data assimilation) and with the HiFi model without data assimilation. We quantify the performance of throttle flow forecasting using the mean absolute error (MAE), Nash-Sutcliffe efficiency (NSE) and persistence index (PI). Accurate forecasts are most important immediately before and during rain events and we therefore quantify the performance of the throttle flow only for time periods with an observed flow higher than $0.05 \mathrm{~m}^{3} / \mathrm{s}$. The overflow forecasting skill is evaluated using the true positive rate (TPR) and positive predictive value (PPV). These evaluation measures are presented in the next sections.

\subsubsection{Mean absolute error}

The MAE is calculated as

$$
\text { MAE }=\frac{\sum\left|Y_{\text {model }}(t)-Y_{\text {obs }}(t)\right|}{n}
$$

411 where $\mathrm{Y}_{\text {model }}(t)$ is the modelled value and $\mathrm{Y}_{\text {obs }}(t)$ is the observed value at time $t$ while $n$ is the number 412 of time steps.

\section{$413 \quad$ 3.6.2 Nash Sutcliffe Efficiency}

414 The NSE is calculated as

$$
\mathrm{NSE}=1-\frac{\sum\left(\mathrm{Y}_{\text {model }}(t)-\mathrm{Y}_{\mathrm{obs}}(t)\right)^{2}}{\sum\left(\mathrm{Y}_{\text {model }}(t)-\overline{\mathrm{Y}_{\mathrm{obs}}}\right)^{2}}
$$

415 where $\overline{Y_{\mathrm{obs}}}$ is the mean value of the observations. NSE is a performance measure, ranging between 0 416 and 1 . Due to the squaring of the terms, it especially punishes large deviations from the model to the 417 observation. An NSE of 1 means a perfect fit between the model and the observation.

\subsubsection{Persistence index}

419 The persistence index (PI) is calculated as

$$
\mathrm{PI}=1-\frac{\sum\left(\mathrm{Y}_{\text {model }}(t)-\mathrm{Y}_{\mathrm{obs}}(t)\right)^{2}}{\sum\left(\mathrm{Y}_{\mathrm{obs}}(t)-\mathrm{Y}_{\mathrm{obs}}(t-j)\right)^{2}}
$$

where $j$ represents the horizon of the prediction. $\mathrm{Y}_{\mathrm{obs}}(t-j)$ is thus the observation at the time of the prediction. PI compares the performance of the model forecast with the performance of the last known observation (Kitanidis and Bras, 1980), often denoted as persistent forecast. If PI $<0$, the persistent forecast (last known observation) is better than the model forecast, and vice versa if PI $>0$. 
We use the true positive rate (TPR) and positive predictive value (PPV) to determine how well the data assimilated model forecasts the observed CSOs. There is no flow meter installed at the CSO outlet and we can therefore only base the CSO evaluation on the measured water level in the overflow structure. Observations above crest level are categorized as 'CSO' and measurements below crest level are categorized as 'no CSO'. The CSO predicted by the surrogate model (updated and baseline) and by the HiFi model are also categorized as CSO/no CSO. This information is used to make a contingency table (Figure 6).

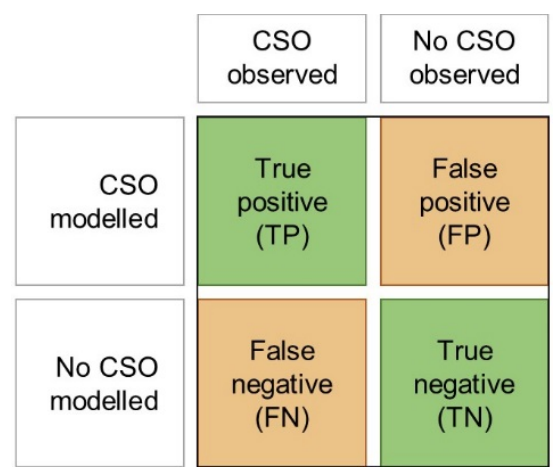

Figure 6. Contingency table.

The true positive rate (TPR) and positive predictive value (PPV) can be calculated based on the contingency table (Saito and Rehmsmeier, 2015):

$$
\begin{aligned}
& \mathrm{TPR}=\frac{\mathrm{TP}}{\mathrm{TP}+\mathrm{FN}} \\
& \mathrm{PPV}=\frac{\mathrm{TP}}{\mathrm{TP}+\mathrm{FP}}
\end{aligned}
$$

436 The TPR shows how many of the observed CSOs are captured by the model prediction. PPV 437 contrarily indicates how much you can trust that a predicted CSO will actually occur. Both TPR and 438 PPV have 0 as the worst outcome and 1 as a perfect score. The two measures will in some cases be 439 counteractive. If the model too often predicts that a CSO will occur it will likely capture all observed 440 CSOs (high TPR) but at the same time the prediction cannot be trusted very much (low PPV). This 441 implies one should look at both measures when evaluating forecast performance.

\section{Results and discussion}

\section{$443 \quad 4.1$ Evaluation of throttle and CSO flow forecast performance}

444 Figure 7 shows the five evaluation measures for each of the four experimental setups (EnKF-Q, 445 EnKF-QV, EnKF-LV and EnKF-LQ). Figure 8 shows the volume of water subtracted or added to 446 each compartment for each time step for the default setups (Table 1). All schemes updated all 447 compartments to some extent. The largest update happened in the downstream compartment for all 448 four setups. For all four default setups there was almost no forecast skill left after 120 minutes, and all 449 updates were "washed out" after 180 minutes. Of the four default setups, EnKF-LV performed the 
450 best in all five evaluation measures for forecast lengths from 15 minutes and above, except for PPV 451 where EnKF-Q and EnKF-LQ were superior. If the goal is to predict CSOs, EnKF-LQ should not be 452 used due to the poor TPR. Also, this experimental setup excelled neither in MAE nor in NSE. The 453 EnKF-LQ setup is seen to have the largest update, but gave the worst forecast skill. Constraining the 454 update may thus increase the performance of this experimental setup.

455 By examining PI in Figure 7 it is seen that from 15 minutes and above it was better to use both the 456 updated surrogate model and baseline surrogate model for forecasting than using the last observation. 457 This was not the case for the one-minute prediction because the flow generally does not vary much 458 from one time step to the next. The PI for the HiFi model shows that it would be better to use the last 459 measurement for forecasting up to 60 minutes ahead, which indicates that the HiFi model has not been 460 properly calibrated for this upstream area. This poor performance of the HiFi model is also seen with 461 respect to MAE, NSE and TPR. The surrogate model was built on the basis of the HiFi model and we 462 believe that this increase in performance must stem from a coincidental favourable simplification of 463 inaccurate descriptions in the HiFi model.

464 The noise specifications of the filter were changed to evaluate the sensitivity of each experimental 465 setup. The worst and best performances for each experimental setup (denoted 'lower and upper 466 bounds') are shown in Figure 7. The values used to make these upper and lower bounds do not 467 necessarily stem from one specific combination of noise formulations for all forecast horizons. From 468 here it is clear that EnKF-QV was very sensitive to the choice of noise parameters, as the upper and 469 lower bounds are far apart. Contrarily, the EnKF-LV bounds are very close together, meaning that this 470 scheme was insensitive to the noise description. This is an advantage in an operational setting, where 471 the time and data are often not available for fine-tuning of the data assimilation. It can also be seen 472 that the results based on the default values were often very close to the upper bounds, meaning that 473 tuning of the noise formulation had very little added benefit. 


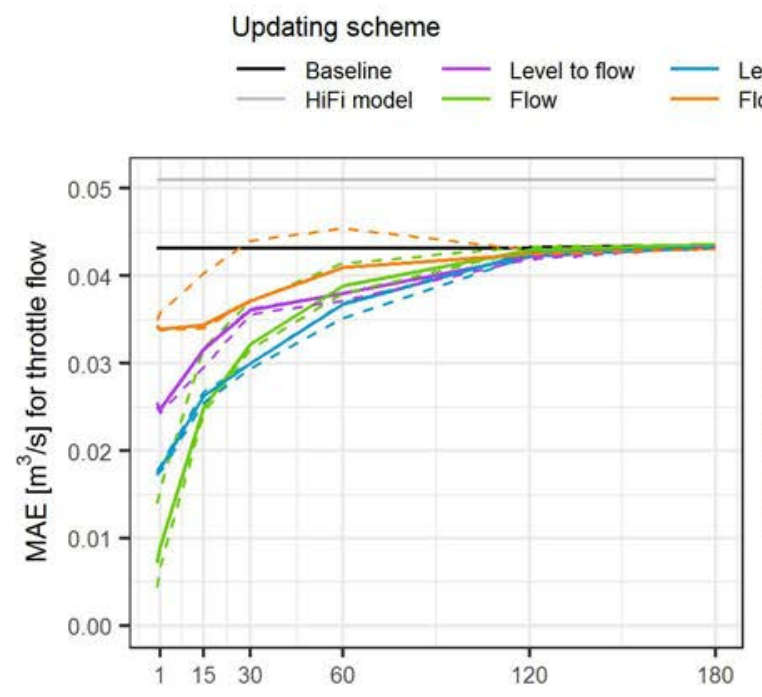

Noise formulation

- HiFi model

Level to flow
Flow

Level to volume
Flow to volume

Default

- - Upper and lower bound
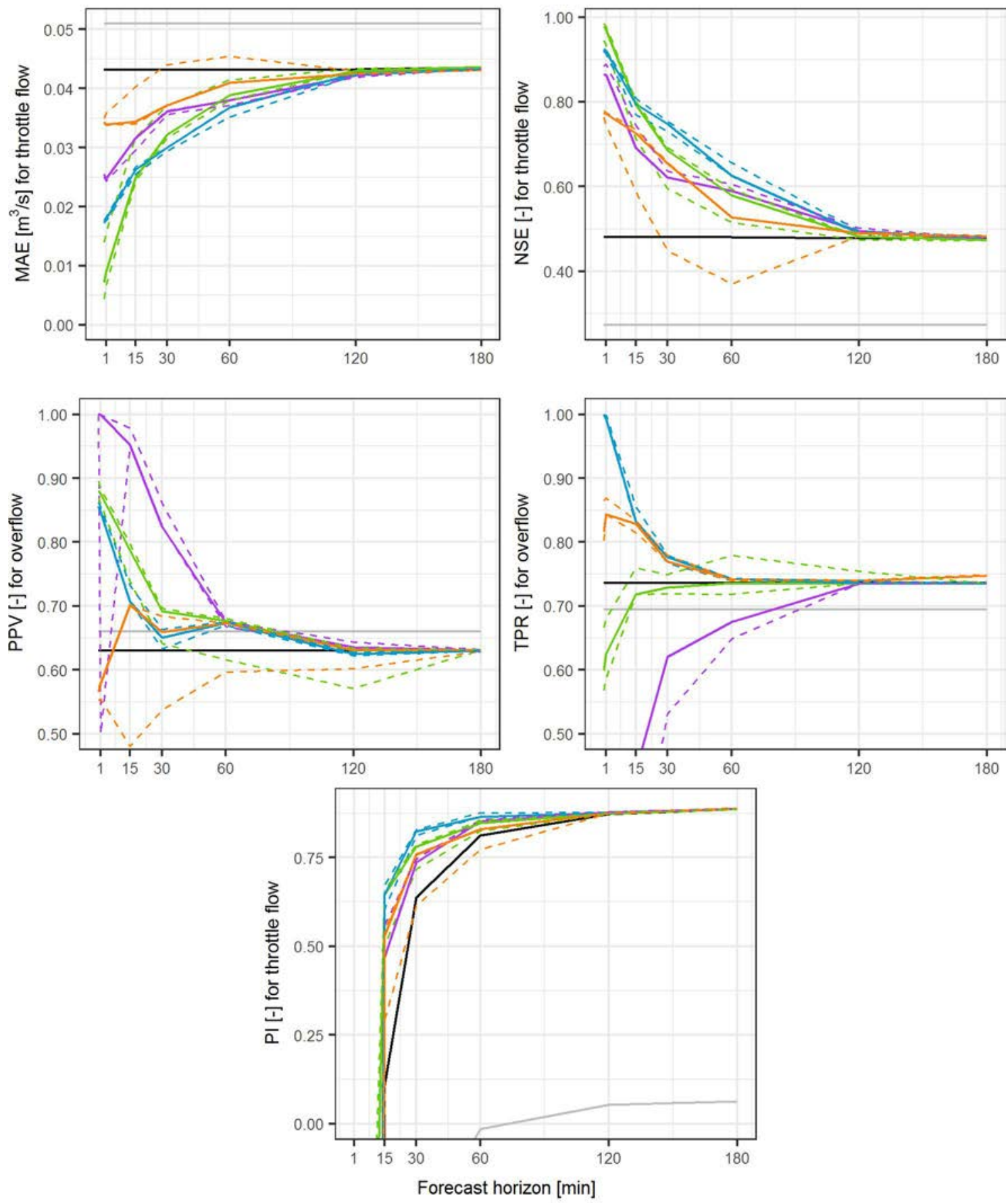

Forecast horizon [min]

Figure 7. Performance comparison between the four experimental setups. MAE, NSE, PPV and TPR takes values at 0, 1, 15, 30, 120 and 180 minutes, whereas PI takes values at 1 minute and above. The default noise formulation represents the noises estimated or calculated from the HiFi model, whereas the upper and lower bound is the worst and best performance for each experimental setup in the sensitivity study. The axes have been scaled for better visualization. 

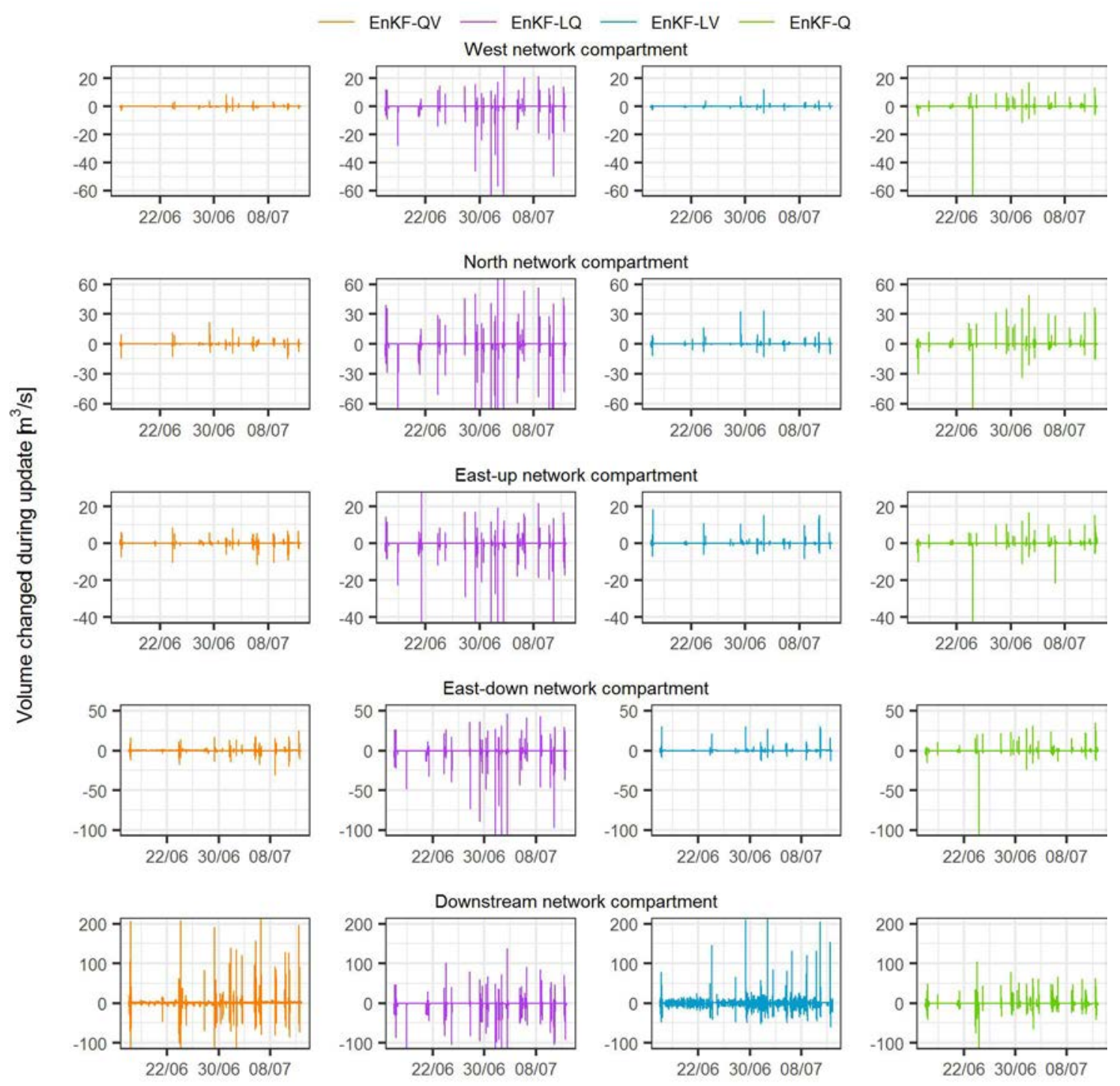

Figure 8. The updated volume in each time step for the four experimental setups. Some values are removed by scaling the $y$ axis to make a better visualization.

\subsection{Throttle and CSO flow forecasts using EnKF-LV for selected events}

Two different events are used to visualize the performance of the update for the best performing (default) experimental setup for both the throttle flow predictions (Figure 9a) and the CSO flow predictions (Figure 9b). The use of five different evaluation measures on six different forecast horizons (1-180 minutes) makes it difficult to give an unambiguous conclusion about which setup is the best, since this will depend on the purpose of the modelling. Do we want to be good overall at capturing the flow (low MAE), are we interested in the peaks (high NSE), do we want to capture the observed CSO events (high TPR), or do we want to be able to have a high confidence in our CSO prediction (high PPV)? Based on an overall assessment, EnKF-LV was chosen as the setup with the best performance.

For the 15-minute forecast, the updated surrogate model captured both the peaks and lows of the throttle flow really well compared to the surrogate baseline and HiFi models. We also see that the 
baseline surrogate and HiFi models in general predicted that the CSO would happen later than it actually did based on the measurements (visualized as CSO/no CSO). The updated surrogate model managed to predict the CSO earlier but was nevertheless still delayed compared to the measurement. This was a general tendency for the entire time series. On four occasions, the updated surrogate model predicted a CSO that was not captured by the baseline surrogate model; one of these is shown in Figure 9b. Neither did the HiFi model predict the CSO in three of these four instances. Only on one occasion did the updated surrogate model completely miss a CSO event.

When looking at the 60-minute forecasts, it is clear that an increased forecast horizon decreased the performance significantly. There was still some forecast skill left for the throttle flow prediction, but the CSO prediction was almost identical for the updated and baseline surrogate models.

The updated surrogate model had a too high DWF compared to the measurements. A potential explanation is that it is difficult to obtain the "true" level-flow relationship in this range, since the uncertainty on the sensors increases for small amounts of water; furthermore, the level-flow relationship can be highly influenced by, for example, sedimentation.

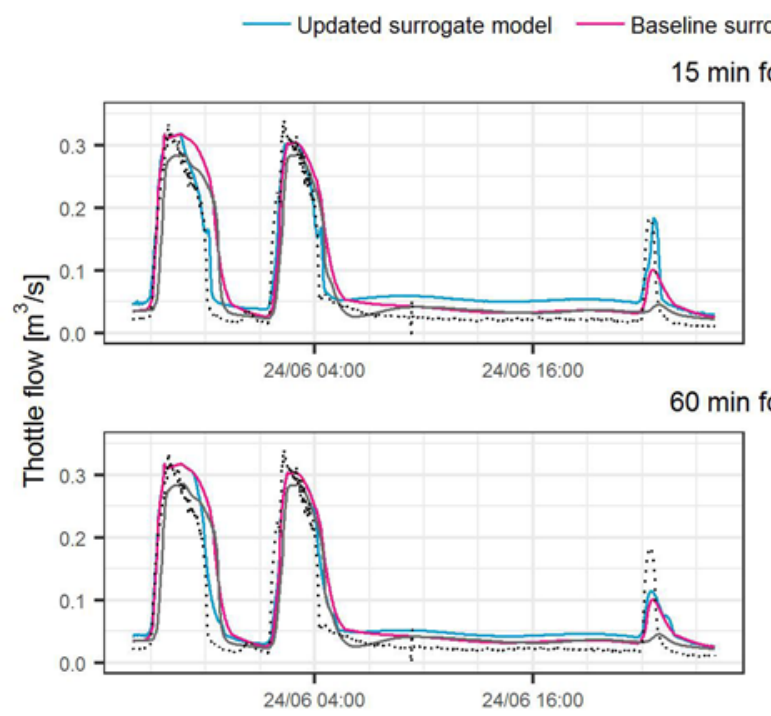

(a) 


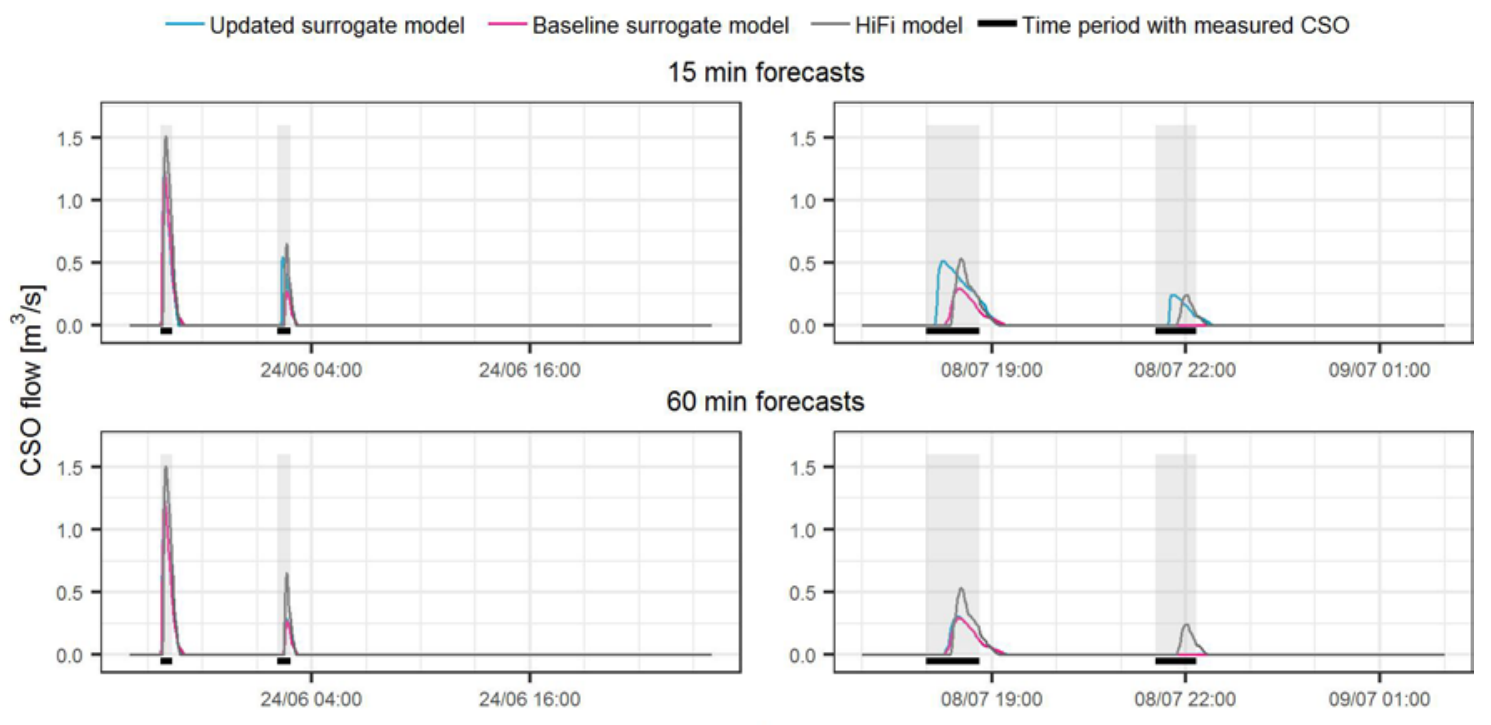

(b)

Figure 9. (a) Throttle flow forecast and (b) CSO flow forecast, for $15 \mathrm{~min}$ (top) and $60 \mathrm{~min}$ (bottom) with the baseline surrogate model, updated surrogate model (EnKF-LV (default)), and HiFi model compared to measurements.

\subsection{Limitations of the study and future perspectives}

The HiFi model performed worse than the baseline surrogate model. To be able to make a fairer comparison between the HiFi model and the updated surrogate model, the HiFi model could be calibrated. Many filter specifications have to be defined when setting up the EnKF, and this paper investigated the sensitivity of some of these. Other filter specifications, which were not tested in this paper, include the length of the temporal correlation in the rainfall perturbation; error distribution types; the choice between additive or proportional error terms; heuristically estimated noise descriptions for the observations; and the subdivision of the rating curves. Other data assimilation filters than the EnKF could also have been used in the study. Due to the small number of elements in the state vector of the surrogate model, even non-ensemble-based methods, such as the Extended Kalman filter, might have worked well. The limited size of the HiFi model implies that we could also have updated this with the EnKF within the given time constraints. This would, however, have entailed numerous other challenges, such as stability issues and how to specify the noise for the many hydraulic variables. We do foresee that typical applications of the surrogate models as forecast models will be for larger systems that would prohibit the use of a HiFi model. The state vector of the surrogate model will here have several hundred elements and the updating will be based on sensor data from multiple locations, in which case the EnKF is computationally very efficient. The main challenge will in this case be the specification of the temporal and spatial errors of the rainfall estimates, since this will determine the correlations throughout the model ensemble that the EnKF uses for the updating. Specified well, the correlations in the error of the rainfall estimates should make it possible to improve the forecasting also in ungauged parts of the model using data from nearby catchments. Nevertheless, it is to be expected that a localization scheme needs to be introduced to dampen long-range corrections arising from, for example, misspecification of the rainfall error. 
536 Furthermore, the effect of a distribution of DWF to the network compartments and the use of 537 distributed rainfall on the updating performance was not investigated due to the limited spatial size of 538 the catchment area.

\section{Conclusions}

540 The low computational speed of HiFi models impedes their use for real-time forecasting in urban 541 drainage systems. Thus, the HiFi model does not pose an alternative to using a surrogate model of a 542 HiFi model for forecasting. Contrarily, conceptual and data-driven models, dependent on data for 543 construction and/or calibration, may be used. However, in the usual situation, where only level data is 544 available, such models will be difficult to construct to produce flow forecasts, and surrogate models 545 are in these cases the only viable solution. Their simplified system description, however, prompts for 546 carrying out data assimilation before forecasting. We therefore constructed a forecast scheme by 547 combining a surrogate model of a distributed urban drainage model with the Ensemble Kalman filter, 548 without relying on historical time series data for model construction and/or calibration. We tested the 549 approach on a small catchment in Copenhagen, Denmark. The model updating was performed with 550 real data by assimilating either water level or flow observations. The predictive skill of the updated surrogate model, the baseline surrogate model and the HiFi model was established by comparing the forecast flows and overflows from a downstream CSO structure to real flow and level observations.

The results showed that the HiFi model would benefit from calibration to the observations used in this study. Updating the surrogate model provides significantly better results than both the baseline surrogate model and the HiFi model. This was valid for forecasts up to two hours ahead when evaluating the models' ability to forecast the flow through the CSO structure's throttle pipe and the CSO discharge, showing that updating can counteract inaccuracies stemming from the HiFi model. Furthermore, updating using water level observations resulted in better flow forecasts than basing the assimilation on flow data - even though the forecasts were validated against flow data from the same flow sensor. Level sensors are the predominant sensor type in urban drainage systems, since these are cheaper and more reliable than flow sensors. It is therefore highly beneficial that the use of level measurements improved the forecast skill more than flow measurements. Another benefit of using water level data for the updating was that the forecast skills were rather insensitive to the noise formulations in the filter, which means that the experimental setup is robust and requires less tuning. This is especially valuable for operational implementations where there may be limited time and data for fine-tuning of the model.

This paper shows that updating of surrogate models may constitute a robust and efficient way of creating both flow and overflow forecasts. The robustness of the method and the use of cheap level sensors are important for the operationalization of data assimilation. Our hope is that this, along with more research on the topic, will make it the norm rather than the exception to update operational, online urban drainage models with measurements in real time. 


\section{Acknowledgements}

573 We would like to thank Francesco Righetti, who constructed the surrogate model for the case area, Peter Steen Mikkelsen and Ole Mark for contributing to the initial concept of the paper and useful discussions, and HOFOR for providing us with the HiFi model and sensor data. This project was supported by Innovation Fund Denmark through the Water Smart Cities Project.

\section{Declarations of interest}

None.

\section{References}

Abaza, M., Garneau, C., Anctil, F., 2015. Comparison of sequential and variational streamflow assimilation techniques for short-term hydrological forecasting. J. Hydrol. Eng. 20.

Angel, S., Parent, J., Civco, D.L., Blei, A., Potere, D., 2011. The dimensions of global urban expansion: Estimates and projections for all countries, 2000-2050. Prog. Plann. 75, 53-107.

Barco, J., Wong, K.M., Stenstrom, M. (2008). Automatic calibration of the U.S. EPA SWMM model for a large urban catchment. Journal of Hydraulic Engineering 134(4), 466-474.

Bertrand-Krajewski, J.L., Bardin, J.P., Mourad, M., Béranger, Y., 2003. Accounting for sensor calibration, data validation, measurement and sampling uncertainties in monitoring urban drainage systems. Water Sci. Technol. 47, 95-102.

Borup, M., Grum, M., Linde, J.J., Mikkelsen, P.S., 2016. Dynamic gauge adjustment of high-resolution Xband radar data for convective rain storms: Model-based evaluation against measured combined sewer overflow. J. Hydrol. 539, 687-699.

Borup, M., Grum, M., Madsen, H., Mikkelsen, P.S., 2014. Updating distributed hydrodynamic urban drainage models. In Proceedings of the $13^{\text {th }}$ International Conference on Urban Drainage, Sarawak, Malaysia, 18.

Borup, M., Grum, M., Mikkelsen, P.S., 2011. Real time adjustment of slow changing flow components in distributed urban runoff models. In Proceedings of the $12^{\text {th }}$ International Conference on Urban Drainage, Porto Alegre, Brazil, 1-8.

Borup, M., Madsen, H., Grum, M., Mikkelsen, P.S., 2018. Technical note on the dynamic changes in Kalman gain when updating hydrodynamic urban drainage models. Geosciences 8, 416.

Borup, M., Thrysøe, C., Arnbjerg-Nielsen, K., Righetti, F., Mikkelsen, P.S., 2017. A fast surrogate model tailor-made for real time control. In Proceedings of the $14^{\text {th }}$ International Conference on Urban Drainage, Prague, Czech Republic, 1-4.

Borup, M., Grum, M., Mikkelsen, P.S., 2013. Comparing the impact of time displaced and biased precipitation estimates for online updated urban runoff models. Water Sci. Technol. 68, 109-116.

Branisavljevic, N., Hutton, C., Kapelan, Z., Vamvakeridou-Lyroudia, L., Savić, D., 2014. Real-time runoff prediction based on data assimilation and model bias reduction. In Proceedings of the $11^{\text {th }}$ International Conference on Hydroinformatics, New York City, USA, 1-8.

Carbajal, J.P., Leitão, J.P., Albert, C., Rieckermann, J., 2017. Appraisal of data-driven and mechanistic emulators of nonlinear simulators: The case of hydrodynamic urban drainage models. Environ. Model. Softw. 92, 17-27.

Clark, M.P., Rupp, D.E., Woods, R.A., Zheng, X., Ibbitt, R.P., Slater, A.G., Schmidt, J., Uddstrom, M.J., 2008. Hydrological data assimilation with the ensemble Kalman filter: Use of streamflow observations to update states in a distributed hydrological model. Adv. Water Resour. 31, 1309-1324.

DHI, 2016. MIKE URBAN. Collection System. Hørsholm, Denmark. 
Evensen, G., 1994. Sequential data assimilation with a nonlinear quasi-geostrophic model using Monte Carlo methods to forecast error statistics. J. Geophys. Res. 99, 10143-10162.

Evensen, G., 2003. The Ensemble Kalman Filter: Theoretical formulation and practical implementation. Ocean Dyn. 53, 343-367.

Hansen, L.S., Borup, M., Møller, A., Mikkelsen, P.S., 2014. Flow forecasting using deterministic updating of water levels in distributed hydrodynamic urban drainage models. Water 6(8), 2195-2211.

Houser, P.R., De Lannoy, G.J.M., Walker, J.P., 2012. Hydrologic data assimilation, in: J. Tiefenbacher (Eds.), Approaches to managing disaster - assessing hazards, emergencies and disaster impacts. Rijeka, Croatia: InTech, pp. 42-64.

Hutton, C.J., Vamvakeridou-Lyroudia, L.S., Kapelan, Z., Savic, D.A., 2010. Real-time modelling and data assimilation techniques for improving the accuracy of model predictions: Scientific report. PREPARED 2011.010 .

Hutton, C.J., Kapelan, Z., Vamvakeridou-Lyroudia, L., Savić, D., 2014. Real-time data assimilation in urban rainfall-runoff models. Procedia Eng. 70, 843-852.

Jørgensen, H.K., Rosenørn, S., Madsen, H., Mikkelsen, P.S., 1998. Quality control of rain data used for urban runoff systems. Wat. Sci. Technol. 37, 113-120.

Kaspersen, P.S., Ravn, N.H., Arnbjerg-Nielsen, K., Madsen, H., Drews, M., 2017. Comparison of the impacts of urban development and climate change in exposing European cities to pluvial flooding. Hydrol. Earth Syst. Sci., 1-26.

Kitanidis, P.K., Bras, R.L., 1980. Real-time forecasting with a conceptual hydrologic model: 2. Applications and results. Water Resour. Res. 16, 1034-1044.

Liu, Y., Weerts, A.H., Clark, M., Hendricks Franssen, H.J., Kumar, S., Moradkhani, H., Seo, D.J., Schwanenberg, D., Smith, P., Van Dijk, A.I.J.M., Van Velzen, N., He, M., Lee, H., Noh, S.J., Rakovec, O., Restrepo, P., 2012. Advancing data assimilation in operational hydrologic forecasting: progresses, challenges, and emerging opportunities. Hydrol. Earth Syst. Sci. 16, 3863-3887.

Lund, N., Borup, M., Halvgaard, R., Falk, A.K., Mark, O., Madsen, H., Mikkelsen, P.S., 2017. Advancing from underground to above-ground model predictive control in urban drainage. In Proceedings of the $14^{\text {th }}$ International Conference on Urban Drainage, Prague, Czech Republic, 1-4.

Lund, N.S.V., Falk, A.K.V., Borup, M., Madsen, H., Mikkelsen, P.S., 2018a. Model predictive control of urban drainage systems: A review and perspective towards smart real-time water management. Crit. Rev. Environ. Sci. Technol. 48, 279-339.

Lund, N., Mazzoleni, M., Madsen, H., Mark, O., Mikkelsen, P.S., Solomatine, D., 2018b. Using the Ensemble Kalman filter to update a fast surrogate model for flow forecasting. In $11^{\text {th }}$ International Conference on Urban Drainage Modelling, Palermo, Italy, 1-5.

Löwe, R., Thorndahl, S., Mikkelsen, P.S., Rasmussen, M.R., Madsen, H., 2014. Probabilistic online runoff forecasting for urban catchments using inputs from rain gauges as well as statically and dynamically adjusted weather radar. J. Hydrol. 512, 397-407.

McMillan, H.K., Hreinsson, E.O., Clark, M.P., Singh, S.K., Zammit, C., Uddstrom, M.J., 2013. Operational hydrological data assimilation with the recursive ensemble Kalman filter. Hydrol. Earth Syst. Sci. 17, 21-38.

Pedersen, J.W., Lund, N.S.V., Borup, M., Löwe, R., Poulsen, T.S., Mikkelsen, P.S., Grum, M., 2016. Evaluation of maximum a posteriori estimation as data assimilation method for forecasting infiltration-inflow affected urban runoff with radar rainfall input. Water 8, 1-15.

Rakovec, O., Weerts, A.H., Hazenberg, P., F. Torfs, P.J.J., Uijlenhoet, R., 2012. State updating of a distributed hydrological model with Ensemble Kalman filtering: Effects of updating frequency and observation network density on forecast accuracy. Hydrol. Earth Syst. Sci. 16, 3435-3449.

Rasmussen, M. R., Thorndahl, S., Schaarup-Jensen, K., 2008. A low cost calibration method for urban drainage models. In Proceedings of the $11^{\text {th }}$ International Conference on Urban Drainage, Edinburgh, Scotland, 1-6.

Refsgaard, J.C., 1997. Validation and intercomparison of different updating procedures for real-time 
Saito, T., Rehmsmeier, M., 2015. The Precision-recall plot is more informative than the ROC plot when evaluating binary classifiers on imbalanced datasets. PLoS One 10.

Thorndahl, S., Poulsen, T.S., Bøvith, T., Borup, M., Ahm, M., Nielsen, J.E., Grum, M., Rasmussen, M.R., Gill, R., Mikkelsen, P.S., 2013. Comparison of short-term rainfall forecasts for model-based flow prediction in urban drainage systems. Water Sci. Technol. 68, 472-478.

Thorndahl, S., Beven, K.J., Jensen, J.B., Schaarup-Jensen, K., 2008. Event based uncertainty assessment in urban drainage modelling, applying the GLUE methodology. J. Hydrol. 357, 421-437.

Thrysøe, C., Arnbjerg-Nielsen, K., Borup, M., 2019. Identifying fit-for-purpose lumped surrogate models for large urban drainage systems using GLUE. J. Hydrol. 568, 517-533.

Tscheikner-Gratl, F., Zeisl, P., Kinzel, C., Leimgruber, J., Ertl, T., Rauch, W., Kleidorfer, M., 2016. Lost in calibration: why people still do not calibrate their models, and why they still should - a case study from urban drainage modelling. Water Sci. Technol. 74(10), 2337-2348.

Wolfs, V., Villazon, M.F., Willems, P., 2013. Development of a semi-automated model identification and calibration tool for conceptual modelling of sewer systems. Water Sci. Technol. 68, 167-175.

681

Zhang, D., Madsen, H., Ridler, M.E., Refsgaard, J.C., Jensen, K.H., 2015. Impact of uncertainty description on assimilating hydraulic head in the MIKE SHE distributed hydrological model. Adv. Water Resour. 86, 400413. 